\title{
Metastatic melanoma: prognostic factors and survival in patients with brain metastases
}

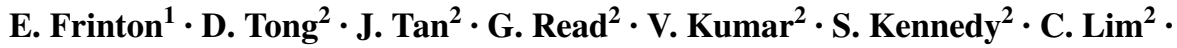 \\ R. E. Board ${ }^{2}(1)$
}

Received: 30 April 2017 / Accepted: 1 August 2017 / Published online: 17 August 2017

(c) The Author(s) 2017. This article is an open access publication

\begin{abstract}
Brain metastases from malignant melanoma carry a poor prognosis. Novel systemic agents have improved overall survival (OS), but the value of wholebrain radiotherapy (WBRT) and stereotactic radiosurgery (SRS) remains uncertain. The melanoma-specific graded prognostic assessment (msGPA) provides useful prognostic information, but the relevance to the modern-day population has not been validated. Since 2011, 53 patients received treatment for brain metastases from malignant melanoma at the Rosemere Cancer Centre medical oncology clinic. Data were collated on demographic factors and survival. Survival analyses were performed using Kaplan-Meier methods. Cox regression was used to identify prognostic factors on univariate and multivariate analysis. OS from the date of diagnosis of brain metastases was 4.83 months (range 0.27-30.4 months). On univariate analysis, BRAF, performance status and msGPA were significant prognostic indicators for OS $(\mathrm{p}=0.0056, \mathrm{p}=0.0039$ and $\mathrm{p}=0.0001$ respectively). msGPA remained significant on multivariate analysis $(\mathrm{p}=0.0006)$. OS for BRAF-positive patients receiving targeted treatment $(n=22)$ was significantly better than for BRAF-negative patients $(n=26)$, with median survival times of 8.2 and 3.7 months respectively ( $\mathrm{p}=0.0039$, HR 2.36). SRS combined with systemic agents $(\mathrm{n}=16)$ produced an OS of 13.5 months. Patients receiving WBRT alone $(n=21)$ had a poor prognosis (2.2 months). The msGPA remains a valid prognostic indicator in the era of novel systemic treatments for melanoma. BRAF-positive patients receiving
\end{abstract}

\footnotetext{
R. E. Board

Ruth.Board@1thtr.nhs.uk

University of Manchester, Manchester, UK

2 Lancashire Teaching Hospitals NHS Trust, Royal Preston Hospital, Preston PR2 9HT, UK
}

targeted agents during their treatment had favorable survival outcomes. WBRT alone should be use with caution in the active management of melanoma brain metastases.

Keywords Metastatic melanoma B Brain metastases · Survival $\cdot$ Radiotherapy

\section{Background}

Malignant melanoma is the fifth most common cancer in the UK. Although the majority of patients present with early stage, operable disease, up to $20 \%$ have metastases at presentation [1]. Brain metastases occur in approximately $44 \%$ patients with metastatic melanoma, with a median overall survival (OS) of just 4 months [2]. Traditionally, brain metastases from melanoma have been deemed incurable by systemic therapy. Poor response rates to chemotherapy are most likely due to low drug concentrations accessing malignant cells owing to the protective nature of the blood-brain barrier [1]. There is evidence for the efficacy of novel systemic agents, both targeted BRAF inhibition and immunotherapy, in brain metastases from melanoma [1, 3-5]. Local treatment options include neurosurgery and radiotherapy. The latter is available for administration in two different forms; stereotactic radiosurgery (SRS) for patients with lowvolume, low count brain metastases [6], or whole brain radiotherapy (WBRT) for patients with more widely disseminated intracranial disease. The evidence base for the efficacy of WBRT in the treatment of melanoma brain metastases is inconclusive. Many studies have shown no improvement in OS [7-9]. It has been hypothesised that this may be due to the resistant nature of melanoma cells to non-SRS radiation, having some ability to repair themselves following radiation insult $[9,10]$. Other analyses suggest that WBRT may 
provide some benefit to intracranial control, particularly when used in combination with SRS and when there is stable extracranial disease [11]. In the absence of definitive guidance, clinicians can utilise a variety of prognostic tools to inform treatment decisions. The melanoma-specific Graded Prognostic Assessment (msGPA) [12] is one such tool that allocates patients into four categories according to number of brain metastases and their Karnofsky performance status (KPS). Patients with a higher msGPA score have a better OS [12]. The relevance of this tool to the modern day patient population, with its access to novel systemic therapies, has not been validated. One criticism of the msGPA is that it does not take into account patient age, presence of extracranial disease, leptomeningeal disease, aggregate brain tumour volume or BRAF status, all of which may be potential important prognostic indicators [2, 4, 13-15].

We aim to assess the validity of the msGPA in the modern day patient population. Important prognostic factors that influence the survival of patients with brain metastases from melanoma will be identified. The efficacy of SRS combined with other systemic therapies versus WBRT will be assessed.

\section{Methods and materials}

Patients undergoing treatment for brain metastases in metastatic melanoma at the Rosemere Cancer Centre, within the Lancashire and South Cumbria Cancer Network (LSCCN), seen in the medical oncology clinic between 2011 and 2016 were identified $(n=57)$. The hospital database system was used to obtain clinic letters, scan results, BRAF testing outcomes and treatment regimes. The information was collated using Microsoft Excel (2010) software. When exact dates were unavailable, the 15 th of the month was used. BRAFmutant positive patients $(n=4)$ who presented prior to the availability of BRAF inhibitors were excluded to enable the efficacy of novel targeted therapies to be assessed.

Treatment of intracranial and extracranial disease was determined by BRAF status, symptoms including performance status, previous treatment, number of brain metastases, patients' preferences and availability and funding of drugs as determined in the UK by the National Institute for Health and Care Excellence and the UK National Cancer Drugs Fund. In 2011 single agent BRAF inhibition was available in the UK as first line treatment, and subsequently in combination with MEK inhibition in July 2016. Second line ipilimumab was available in 2011 and first line in 2014. PD1 antibodies were available from the end of 2015 in both untreated and previously treated patients. Combination immunotherapy was not available during the time period of this audit nor were any clinical trials available for patients with brain metastases. Patients' history and radiology were discussed at both a specialist skin and brain multidisciplinary team meeting to determine suitability for neurosurgery and radiotherapy including stereotactic treatment. Generally, patients with multiple brain metastases from BRAF positive melanoma would be offered BRAF inhibition $( \pm$ MEK inhibition) as first line, followed by either systemic immunotherapy or whole brain radiotherapy second line. Patients with BRAF negative melanoma would be assessed for surgery and/or stereotactic radiotherapy as first line treatment and then offered systemic immunotherapy if available and not received previously. Patients ineligible for surgery or stereotactic radiotherapy and those patients who had exhausted previous systemic treatment options were assessed for whole brain radiotherapy.

StatsDirect [16] software was used to perform survival analysis according to BRAF status, msGPA score and radiotherapy modality. Kaplan-Meier (KM) curves [17] were generated and tested for statistical significance using peto's log rank [18] technique. Where confidence intervals (CI) are quoted, Andersens 95\% CI are used. Survival is quoted in months, standardised to a 30 day period to allow comparison.

Univariate and multivariate analysis were performed using Cox regression [19]. Prognostic factors tested included age, sex, BRAF status, KPS, msGPA, presence of extra cranial disease and presence of neurological symptoms. Factors that showed statistical significance $(\mathrm{p}<0.05)$ on univariate testing were run in multivariate analysis in various combinations to determine if these associations maintained statistical significance when other factors were taken into account.

\section{Results}

In total, $\mathrm{N}=53$ patients were eligible for analysis following exclusion of BRAF-mutant positive patients who did not receive targeted inhibition. For survival analysis according to BRAF status, a further five patients were excluded due to unknown BRAF status. Basic demographic and clinical covariate data is shown in Table 1 .

Median OS from diagnosis of brain metastases to death was 4.83 months (range 0.27-30.4 months). BRAF-mutant positive patients had significantly better survival times than the BRAF-mutant negative group, at 8.23 month median OS (95\% CI 3.62-12.84) and 3.7 months (95\% CI 2.78-4.62) respectively $(\mathrm{p}=0.0039)$ from the time of diagnosis of brain metastases (Fig. 1). $\mathrm{N}=17$ (32\%) patients were receiving either immunotherapy or BRAF inhibition at the time of their brain metastases diagnosis. The median number of cycles of systemic treatment until development of brain metastases was 5 (range $=1-21) . \mathrm{N}=6$ patients $(35 \%)$ continued on the same systemic treatment after their brain 
Table 1 Patient demographics

\begin{tabular}{|c|c|}
\hline Total number of patients & 53 \\
\hline Age, median (range) & $61(20-85)$ \\
\hline \multicolumn{2}{|l|}{ Sex, n $(\%)$} \\
\hline Male & $31(58)$ \\
\hline Female & $22(42)$ \\
\hline \multicolumn{2}{|l|}{ BRAF status, n (\%) } \\
\hline Positive & $25(47)$ \\
\hline Negative & $22(42)$ \\
\hline Unknown & $5(9)$ \\
\hline $\begin{array}{l}\text { Brain metastases present at initial diagnosis of meta- } \\
\text { static melanoma, } \mathrm{n}(\%)\end{array}$ & $22(42)$ \\
\hline Brain metastases symptomatic, $\mathrm{n}(\%)$ & $41(77)$ \\
\hline \multicolumn{2}{|l|}{ Number of brain metastases, $\mathrm{n}(\%)$} \\
\hline 1 & $15(28)$ \\
\hline $2-3$ & $10(19)$ \\
\hline$>3$ & $28(53)$ \\
\hline \multicolumn{2}{|l|}{ KPS, n (\%) } \\
\hline $90-100$ & $24(45)$ \\
\hline $70-80$ & $21(40)$ \\
\hline$<70$ & $8(15)$ \\
\hline \multicolumn{2}{|l|}{ msGPA score, $\mathrm{n}(\%)$} \\
\hline $0-1$ & $21(40)$ \\
\hline 2 & $12(23)$ \\
\hline 3 & $6(11)$ \\
\hline 4 & $14(26)$ \\
\hline Extra cranial disease presence, $\mathrm{n}(\%)$ & $42(79)$ \\
\hline \multicolumn{2}{|l|}{ Treatment for brain metastases, $\mathrm{n}(\%)$} \\
\hline Total Neurosurgery & $9(17)$ \\
\hline Neurosurgery alone & $3(6)$ \\
\hline Total SRS & $8(15)$ \\
\hline SRS alone & $3(6)$ \\
\hline Total WBRT & $23(43)$ \\
\hline WBRT alone & $17(32)$ \\
\hline Total Immunotherapy & $9(17)$ \\
\hline Immunotherapy alone & $2(4)$ \\
\hline Total Targeted BRAF inhibitor & $14(26)$ \\
\hline Targeted BRAF inhibitor alone & $8(15)$ \\
\hline Combination therapy & $15(28)$ \\
\hline Neurosurgery + WBRT & $2(4)$ \\
\hline Neurosurgery + targeted BRAF inhibitor & $4(8)$ \\
\hline Neurosurgery + SRS + immunotherapy & $2(4)$ \\
\hline SRS + immunotherapy & $3(6)$ \\
\hline WBRT + targeted BRAF inhibitor & $2(4)$ \\
\hline WBRT + immunotherapy & $2(4)$ \\
\hline Best supportive care & $5(9)$ \\
\hline
\end{tabular}

metastases were diagnosed. The remainder were switched to an alternative.

SRS was often given in combination with other treatments such as surgery and immunotherapy. $\mathrm{N}=14(26 \%)$ patients were treated with SRS for their brain metastases,

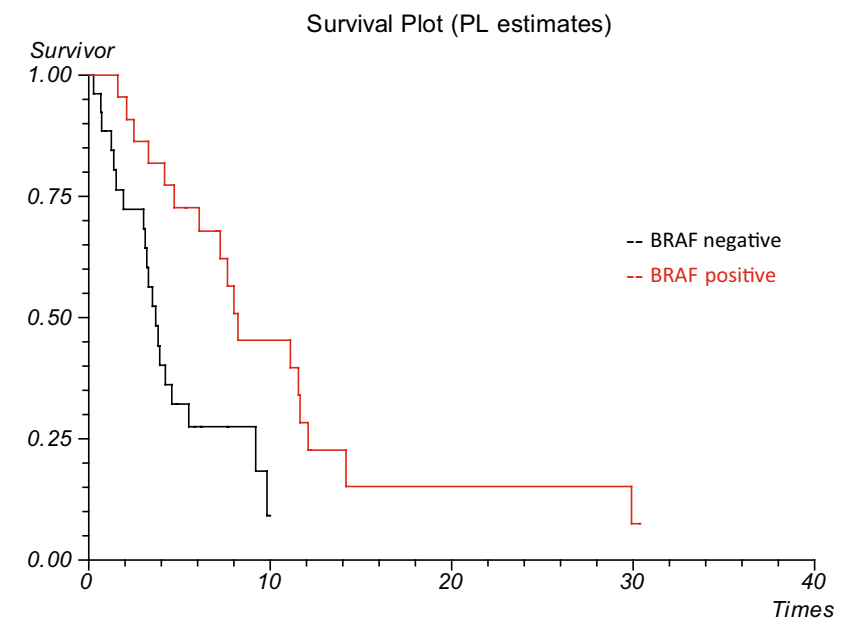

Fig. 1 Overall survival from date of brain metastases diagnosis according to BRAF status. Better survival $(\mathrm{p}=0.0039)$ in the BRAFpositive treated subgroup $(n=25)$, with a median OS of 8.23 months (95\% CI 3.62-12.84) in comparison to 3.7 months (95\% CI 2.784.62) for the BRAF-negative patients $(n=22)$

either initially $(n=9)$ or on progression $(n=5) . N=10$ (71\%) patients demonstrated response to this treatment on radiological follow up. Equal numbers of BRAF-positive and BRAF-negative patients showed response rates to SRS. The remaining patients showed either brain progression or died before follow up. The majority of patients treated with WBRT alone died within 2 months of treatment, so radiological follow up was not available to assess treatment responses.

When compared with WBRT there was a statistically significant improvement in survival in patients suitable for SRS. Patients treated with SRS and/or surgery and/or systemic treatment $(n=16)$ had a median survival of 13.5 months (95\% CI 5.93-21.1) compared to 2.2 months (95\% CI $1.55-2.85)$ in the WBRT group $(n=21)$. This difference reached statistical significance (HR 3.97, $\mathrm{p}=0.0009)$.

Figure 2 shows survival outcomes based on msGPA score. Median survival was 11.6 months for those with a maximum GPA score of $4(\mathrm{n}=15,95 \%$ CI 9.29-13.84), 7.2 months for GPA 3 ( $n=6,95 \%$ CI 6.9-7.57), 4.2 months for GPA $2(n=12,95 \%$ CI 2.36-5.98) and 3.3 months for GPA 0-1 ( $n=20,95 \%$ CI 2.47-4.07). This suggests that the msGPA provides a reliable indication of likely prognosis, with those scoring more highly having better survival outcomes (peto's log rank test, $\mathrm{p}<0.0001$ ).

On univariate analysis, factors shown to be predictive of OS were BRAF status, KPS and msGPA score (msGPA 0-2 vs. msGPS 3-4) (Table 2). Age, sex, presence of neurological symptoms and presence of extra cranial disease were not predictors of survival on univariate analysis. Only msGPA score maintained significance on multivariate analysis (Table 3). 


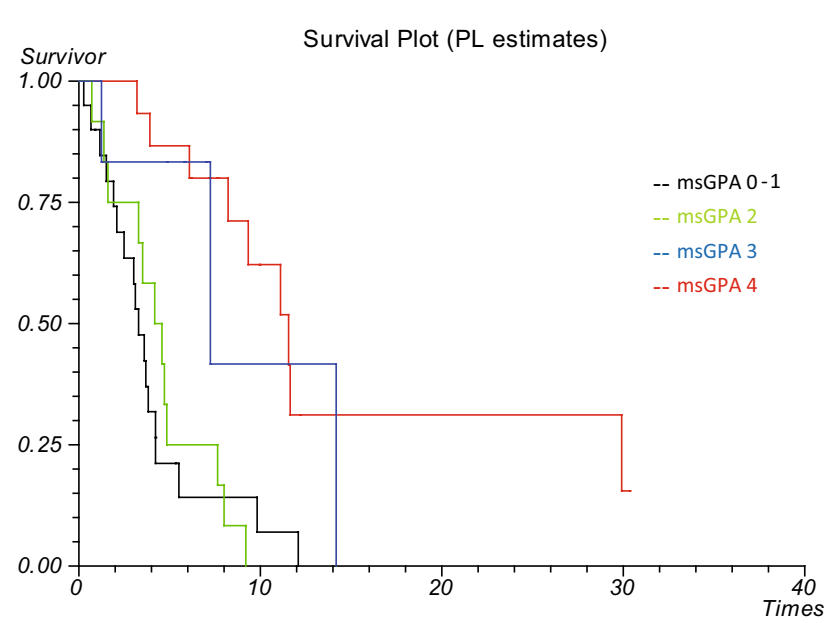

Fig. 2 Overall survival from date of brain metastases diagnosis according to melanoma specific graded prognostic assessment score

Table 2 Univariate analysis of prognostic factors

\begin{tabular}{|c|c|c|c|}
\hline & Hazard ratio & $95 \% \mathrm{CI}$ & p-value \\
\hline \multicolumn{4}{|l|}{ BRAF status } \\
\hline Positive & 0.345 & $0.163-0.732$ & 0.0056 \\
\hline Negative & 1 & & \\
\hline \multicolumn{4}{|l|}{ msGPA score } \\
\hline $0-2$ & 1 & & \\
\hline $3-4$ & 0.194 & $0.0882-0.428$ & $<0.0001$ \\
\hline \multicolumn{4}{|c|}{ Neurological symptoms } \\
\hline Present & 1.85 & $0.701-4.88$ & 0.2141 \\
\hline Absent & 1 & & \\
\hline Age & 1.022 & $0.996-1.048$ & 0.0928 \\
\hline \multicolumn{4}{|l|}{ Sex } \\
\hline Male & 1 & & \\
\hline Female & 0.513 & $0.261-1.012$ & 0.0541 \\
\hline \multicolumn{4}{|l|}{ KPS } \\
\hline $70-100$ & 1 & & \\
\hline$<70$ & 4.003 & $1.56-10.26$ & 0.0039 \\
\hline \multicolumn{4}{|l|}{ Extra cranial disease } \\
\hline Present & 1 & & \\
\hline Absent & 0.912 & $0.397-2.10$ & 0.8303 \\
\hline \multicolumn{4}{|c|}{ Development of brain mets } \\
\hline $\begin{array}{l}\text { Presentation } \\
\text { of metastatic } \\
\text { disease }\end{array}$ & 0.698 & $0.356-1.37$ & 0.2941 \\
\hline $\begin{array}{l}\text { During treatment } \\
\text { for metastatic } \\
\text { disease }\end{array}$ & 1 & & \\
\hline
\end{tabular}

\section{Discussion}

This retrospective study confirms the overall poor prognosis of patients with brain metastases from melanoma. However,
Table 3 Multivariate analysis of prognostic factors

\begin{tabular}{llll}
\hline & Hazard ratio & $95 \%$ CI & p-value \\
\hline $\begin{array}{l}\text { BRAF status } \\
\text { Positive }\end{array}$ & 0.544 & $0.247-1.195$ & 0.1294 \\
Negative & 1 & & \\
msGP & & & \\
$0-2$ & 1 & $0.105-0.539$ & 0.0006 \\
$3-4$ & 0.238 & & \\
KPS & & & \\
$70-100$ & 1 & $0.832-5.704$ & 0.1131 \\
\hline 70 & 2.178 & & \\
\hline
\end{tabular}

it highlights certain treatment options that may be helpful such as BRAF inhibitors in BRAF mutated melanoma and SRS alone or combined with systemic treatment for suitable patients. The ability of the msGPA to accurately discriminate prognostic groupings, and thus its suitability for continued use in a modern population with access to novel treatments, is confirmed. And importantly we recommend that if WBRT alone is the only treatment option available for a particular patient this should be offered only after careful consideration and discussion with the patient as there is a very poor outlook in this group of patients.

We report a median OS from brain metastases diagnosis of 4.83 months. This is comparable other reports in the literature [4]. Patients in our cohort were almost equally distributed between mutant-positive $(n=25)$ and mutantnegative $(n=22)$ categories. This proportion is higher than commonly quoted in the literature, where BRAF mutantpositive melanomas are reported to comprise around $40 \%$ of the total melanoma population [20]. This adds weight to the growing evidence base demonstrating that BRAF-positive melanoma patients are at increased risk of development of brain metastases [21-23].

In our series the BRAF-mutant positive patients had significantly better survival times than the BRAF-mutant negative group, at 8.23 month median OS (95\% CI 3.62-12.84) and 3.7 months (95\% CI 2.78-4.62) respectively $(\mathrm{p}=0.0039)$ from the time of diagnosis of brain metastases. This is due to the benefit of BRAF inhibitors as a treatment for brain metastases in BRAF mutation positive melanoma. The OS of our group of BRAF positive patients compares favorably to those treated in the original BREAK-MB trial, which documented survival times of up to 7.7 months [24]. Thus we provide evidence to support the efficacy of targeted therapies in producing improved survival outcomes in 'real life' BRAF-positive patients. BRAF status was an independent predictor of survival in univariate analysis, in concordance with previous documentation in the literature [2, 21]. It may have potential to be incorporated into prognostic modelling for brain metastases. 
We assessed the use of radiotherapy for the treatment of brain metastases from metastatic melanoma; SRS combined with other systemic treatments and WBRT alone. We found evidence to support the efficacy of SRS. N $=16$ (30\%) patients received SRS for up to three small volume intracerebral metastases, either at first presentation of brain disease or on progression. The median survival time from treatment was 13.5 months (95\% CI 5.93-21.1). This is consistent with previous literature, demonstrating increased intracranial control, OS and the ability to use multiple courses of SRS on disease progression [13-15, 25]. It has been hypothesised that BRAF-mutant positive patients may have increased responsiveness to SRS [26]. However, we found no difference in response rates according to BRAF status.

Evidence for the role of WBRT in local treatment of brain metastases from melanoma is less consistent. Many studies report no benefit to overall survival [7-9], although some neurological symptoms palliation may be offered for symptomatic patients. Others suggest that certain circumstances, such as stable extra-cranial disease or adjuvant treatment with SRS or neurosurgery, may enable WBRT to control intracranial disease for a limited period of time [11]. In our cohort, 21 patients underwent WBRT as the sole treatment for their brain metastases. Median OS was poor at just 2.2 months (95\% CI 1.55-2.85). This may partially be due to an underlying selection bias for a patient population with particularly poor prognoses since WBRT was often offered to those who had exhausted systemic treatment options with multiple disseminated lesions as a palliative measure. It brings into question the appropriateness of recommending this treatment where little survival benefit must be balanced against a potential side effect profile that includes alopecia, neurocognitive decline and fatigue.

We found evidence to support the continued use of the msGPA in the modern day population with use of novel therapies. Our cohort was well distributed between the four prognostic groupings. The scoring system discriminated prognosis well, with a median survival of 11.6 months (95\% CI 9.29-13.84) for those with a maximum GPA of 4 , contrasted to a median survival of 3.3 months $(95 \%$ CI 2.47-4.07) for those with a GPA score of 0-1. The log rank test demonstrated statistical significance in median survival outcomes between the four groupings $(\mathrm{p}<0.0001)$. Our results are similar to those reported in the original Sperduto et al. study of 3.38, 4.7, 8.8 and 13.2 months with increasing msGPA score [12]. Furthermore, the msGPA demonstrated significance when tested on both univariate $(p<0.0001)$ and multivariate analysis $(\mathrm{p}=0.0006)$ in our cohort.

We believe that our patients, recruited from 2011 to 2016, with access to novel systemic therapies, is representative of the current modern day population and practice.
Thus our data demonstrates that the msGPA score is still important in modern day practice, and is able to provide patients with a reliable indication of likely prognosis.

Our results must be interpreted in the light of several limitations; firstly, the retrospective nature of the study meant that KPS scores (and therefore msGPA calculations) along with the precise number and volume of brain metastases may be less reliable. The rapidly changing landscape of melanoma treatments over recent years means that not all the patients in our sample had access to all the treatment options in the same sequence. For example, during the period studied PD1 inhibitors were available in the second line setting only initially and then subsequently as first line. The sample size of 53 patients limits the power of the study to produce statistically significant results applicable outside our cohort. We suggest that further studies of similar design, on a larger cohort, be executed to test the validity of our observations.

\section{Conclusion}

Our study has provided evidence to validate the use of the msGPA in the modern patient population, with its access to novel treatments. The system provides clinicians with an indication of prognosis and may aid patient centered treatment discussions. We have demonstrated that BRAF positive patients receiving targeted treatment have significantly better survival than their BRAF negative counterparts. We were able to evidence the efficacy of SRS in the local treatment of brain metastases irrespective of BRAF status. By contrast, the suitability of WBRT as a standalone management option has been called in to question. Patients unsuitable for SRS who therefore received WBRT had a very poor outcome.

\section{Compliance with ethical standards}

Conflict of interest The authors declare they have no conflict of interest.

Ethical approval and Informed Consent All procedures performed in studies involving human participants were in accordance with the ethical standards of the institutional and/or national research committee and with the 1964 Helsinki declaration and its later amendments or comparable ethical standards. This study was registered with the trust audit department. For this type of study formal consent is not required.

Open Access This article is distributed under the terms of the Creative Commons Attribution 4.0 International License (http://creativecommons.org/licenses/by/4.0/), which permits unrestricted use, distribution, and reproduction in any medium, provided you give appropriate credit to the original author(s) and the source, provide a link to the Creative Commons license, and indicate if changes were made. 


\section{References}

1. Murrell J, Board R (2013) The use of systemic therapies for the treatment of brain metastases in metastatic melanoma: opportunities and unanswered questions. Cancer Treat Rev 39:833-838

2. Davies MA, Liu P, McIntyre S et al [2011]. Prognostic factors for survival in melanoma patients with brain metastases. Cancer 117:1687-1696

3. Margolin K, Ernstoff MS, Hamid O, Lawrence D, McDermott D, Puzanov I, Wolchok JD, Clark JI, Sznol M, Logan TF, Richards J, Michener T, Balogh A, Heller KN, Hodi FS (2012) Ipilimumab in patients with melanoma and brain metastases: an open-label, phase 2 trial. Lancet Oncol 13(5):459-465

4. Wilkins A, Furness A, Corbett RW, Bloomfield A, Porta N, Morris S, Ali Z, Larkin J, Harrington K 2015. The melanoma-specific graded prognostic assessment does not adequately discriminate prognosis in a modern population with brain metastases from malignant melanoma. Br J Cancer 113(9):1275-1281

5. Dummer R, Goldinger SM, Turtschi CP, Eggmann NB, Michielin O, Mitchell L, Veronese L, Hilfiker PR, Felderer L, Rinderknecht JD (2014) Vemurafenib in patients with BRAF(V600) mutationpositive melanoma with symptomatic brain metastases: final results of an open-label pilot study. Eur J Cancer 50(3):611-621

6. Bhatnagar AK, Kondziolka D, Lunsford LD, Flickinger JC (2007) Recursive partitioning analysis of prognostic factors for patients with four or more intracranial metastases treated with radiosurgery. Technol Cancer Res Treat 6(3):153-160

7. Mori Y, Kondziolka D, Flickinger JC, Kirkwood JM, Agarwala S, Lunsford LD (1998). Stereotactic radiosurgery for cerebral metastatic melanoma: factors affecting local disease control and survival. Int J Radiat Oncol Biol Phys 42:581-589

8. Yu C, Chen JC, Apuzzo ML, O'Day S, Giannotta SL, Weber JS, Petrovich Z (2002) Metastatic melanoma to the brain: prognostic factors after gamma knife radiosurgery. Int J Radiat Oncol Biol Phys 52:1277-1287

9. Chang EL, Selek U, Hassenbusch SJ 3rd, Maor MH, Allen PK, Mahajan A, Sawaya R, Woo SY (2005) Outcome variation among "radioresistant" brain metastases treated with stereotactic radiosurgery. Neurosurgery 56:936-945 (discussion 936-945)

10. Doss LL, Memula N (1982) The radioresponsiveness of melanoma. Int J Radiat Oncol Biol Phys 8(7):1131-1134

11. Dyer MA, Arvold ND, Chen YH, Pinnell NE, Mitin T, Lee EQ, Hodi FS, Ibrahim N, Weiss SE, Kelly PJ, Floyd SR, Mahadevan A, Alexander BM (2014) The role of whole brain radiation therapy in the management of melanoma brain metastases. Radiat Oncol 9:143

12. Sperduto PW, Berkey B, Gaspar LE, Mehta M, Curran W (2008). A new prognostic index and comparison to three other indices for patients with brain metastases: an analysis of 1,960 patients in the RTOG database. Int J Radiat Oncol Biol Phys 70(2):510-514

13. Marcus DM, Lowe M, Khan MK, Lawson DH, Crocker IR, Shelton JW, Melton A, Maynard N, Delman KA, Carlson GW, Rizzo M (2014) Prognostic factors for overall survival after radiosurgery for brain metastases from melanoma. Am J Clin Oncol 37(6):580-584

14. Hauswald H, Stenke A, Debus J, Combs SE (2015) Linear accelerator-based stereotactic radiosurgery in 140 brain metastases from malignant melanoma. BMC Cancer 15:537
15. Shultz DB, Modlin LA, Jayachandran P, Von Eyben R, Gibbs IC, Choi CY, Chang SD, Harsh GR, Li G, Adler JR, Hancock SL, Soltys SG (2015). Repeat courses of stereotactic radiosurgery (SRS), deferring whole-brain irradiation, for new brain metastases after initial SRS. Int J Radiat Oncol Biol Phys 92(5):993-999

16. StatsDirect Ltd. (2013) StatsDirect statistical software. StatsDirect Ltd., England. http://www.statsdirect.com

17. Kaplan E, Meier P (1958) Nonparametric estimation from incomplete observations. J Am Stat Assoc 53(282):457-481. Retrieved from http://www.jstor.org/stable/2281868

18. Peto R, Pike MC, Armitage P, Breslow NE, Cox DR, Howard SV, Mantel N, McPherson K, Peto J, Smith PG (1977) Design and analysis of randomized clinical trials requiring prolonged observation of each patient. II. Analysis and examples. Br J Cancer 35(1):1-39

19. Cox D (1972) Regression models and life-tables. J R Stat Soc 34(2):187-220. Retrieved from http://www.jstor.org/ stable/2985181

20. Rubinstein JC, Sznol M, Pavlick AC, Ariyan S, Cheng E, Bacchiocchi A, Kluger HM, Narayan D, Halaban R (2010) Incidence of the V600K mutation among melanoma patients with BRAF mutations, and potential therapeutic response to the specific BRAF inhibitor PLX4032. J Transl Med 8:67

21. Ramanujam S, Schadendorf D, Long GV (2015) Systemic therapies for melanoma brain metastases: which drug for whom and when? Chin Clin Oncol 4(2):25

22. Colombino M, Capone M, Lissia A, Cossu A, Rubino C, De Giorgi V, Massi D, Fonsatti E, Staibano S, Nappi O, Pagani E, Casula M, Manca A, Sini M, Franco R, Botti G, Caracò C, Mozzillo N, Ascierto PA, Palmieri G 2012. BRAF/NRAS mutation frequencies among primary tumors and metastases in patients with melanoma. J Clin Oncol 30(20):2522-2529

23. Capper D, Berghoff AS, Magerle M, Ilhan A, Wöhrer A, Hackl M, Pichler J, Pusch S, Meyer J, Habel A, Petzelbauer P, Birner P, von Deimling A, Preusser M (2012) Immunohistochemical testing of BRAF V600E status in 1,120 tumor tissue samples of patients with brain metastases. Acta Neuropathol 123(2):223-233

24. Long GV, Trefzer U, Davies MA, Kefford RF, Ascierto PA, Chapman PB, Puzanov I, Hauschild A, Robert C, Algazi A, Mortier L, Tawbi H, Wilhelm T, Zimmer L, Switzky J, Swann S, Martin AM, Guckert M, Goodman V, Streit M, Kirkwood JM, Schadendorf D (2012) Dabrafenib in patients with Val600Glu or Val600Lys BRAF-mutant melanoma metastatic to the brain (BREAK-MB): a multicentre, open-label, phase 2 trial. Lancet Oncol 13(11):1087-1095

25. Lwu S, Goetz P, Monsalves E, Aryaee M, Ebinu J, Laperriere N, Menard C, Chung C, Millar BA, Kulkarni AV, Bernstein M, Zadeh G (2013) Stereotactic radiosurgery for the treatment of melanoma and renal cell carcinoma brain metastases. Oncol Rep 29(2):407-412

26. Gallaher IS, Watanabe Y, DeFor TE, Dusenbery KE, Lee CK, Hunt MA, Lin HY, Yuan J (2016) BRAF mutation is associated with improved local control of melanoma brain metastases treated with gamma knife radiosurgery. Front Oncol 6:107 